\title{
Rôle potentiel d'un RGTA dans le recrutement des précurseurs d'ostéoclastes sur un modèle de résorption osseuse synchronisée
}

\section{Potential role of a RGTA in the recruitment of osteoclast precursors in a synchronized model of bone resorption}

JULIEN SIMON-TERMINI, PHILIPPE DOUCET, PHILIPPE LESCLOUS, JEAN-LOUIS SAFFAR

Travail financé par une bourse de recherche allouée par la Société Francophone de Médecine Buccale et Chirurgie Buccale

\begin{abstract}
RÉSUMÉ
Objectif : Les RGTA (ReGenraTing Agents), mimétiques des héparanes sulfates, ont d'importantes propriétés anti-inflammatoires. Ils réduisent le recrutement des précurseurs des cellules effectrices de la résorption osseuse, les ostéoclastes. L'objectif de cette étude est de caractériser, sur un modèle animal de remaniement osseux synchronisé, l'action d'un RGTA sur la sortie vasculaire des leucocytes et des cellules de la lignée monocytaire dont sont issus les précurseurs des ostéoclastes.

Méthode : 33 rats males Wistar ont subi l'extraction de leurs molaires maxillaires droites entraînant une vague de remaniement osseux synchronisé dans le secteur mandibulaire antagoniste. Une injection intra-péritonéale d'un RGTA a été pratiquée 10 heures, puis 11 heures après l'induction du modèle. Les leucocytes ont été quantifiés à l'aide d'une coloration au bleu de toluidine et les cellules monocytaires grâce à leur activité ED1+ en immunohistochimie.

Résultats : Le nombre des monocytes ED1+, recrutés par le site inflammatoire, diminue de moitié chez les animaux traités au RGTA à 10 heures et à 11 heures $(p<0.02$ et $p<0.01)$. En revanche, le nombre des leucocytes totaux recrutés ne se trouve pas diminué de façon significative par le traitement.

Conclusion : Le recrutement des futurs préostéoclastes est diminué de façon significative par l'action du RGTA. Cette diminution est plus marquée quand l'injection a lieu 10 heures après l'induction du modèle. Le recrutement de ces cellules démarre donc rapidement après la dégranulation des mastocytes qui a lieu 9 heures après les extractions.(Med Buccale Chir Buccale 2008; 14 : 161-166)
\end{abstract}

mots clés : RGTA, ostéoclaste, monocyte, leucocyte, résorption osseuse

\section{SUMMARY}

Objective: RGTA (ReGenraTing Agents), polymers engeneered to mimic some of the protective properties of heparanes sulfates, are potent anti-inflammatory agents. These compounds decrease recruitment of osteoclast precursors. The aim of this study was to investigate whether a RGTA is involved in the extravasation of leucocytes and monocyte-commited cells, lineage at the origin of the osteoclast precursors. 
Method: 33 male Wistar rats were used. The right maxillary molars were extracted to induce a wave of synchronized bone remodeling along the buccal cortex of the antagonist mandibular jaw. An intraperitoneal injection of a RGTA was released either 10 hours, either 11 hours after the model was induced. Leucocytes were counted on toluidine blue-stained sections and monocytes were distinguished with their ED1+ activity in immunochemistry.

Results: The number of monocyte ED1+ cells is half decreased with the RGTA treatment at 10 and at 11 hours $(p<0.02$ et $p<0.01)$, whether the number of leucocytes was not significantly affected by this treatment.

Conclusion: Recruitment of cells considered as circulating osteoclastic precursors is strongly decreased by the RGTA. This is more obvious with the 10 hours RGTA treatment. Recruitment of these cells is early after mast cells degranulation which is observed 9 hours after teeth extraction. (Med Buccale Chir Buccale 2008; 14 : 161-166).

key words: RGTA, osteoclast, monocyte, leucocyte, bone resorption

\section{INTRODUCTION}

médecine

buccale

chirurgie

buccale

VOL. $14, \mathrm{~N}^{\circ} 3$ 2008

page 162
Dans un modèle d'inflammation parodontale où la résorption de l'os alvéolaire dépend des ostéoclastes, il a été montré que des molécules mimétiques des héparanes sulfates, les RGTA (ReGeneraTing Agents) ont des propriétés antiinflammatoires importantes [1]. En particulier, ces molécules réduisent très fortement le recrutement des précurseurs des ostéoclastes et agissent sur leur différenciation (ostéoclastes de petite taille) [2]. Les RGTA sont des polymères synthétisés pour mimer les effets protecteurs et réparateurs des héparanes sulfates envers les tissus lésés. Ces propriétés sont dues à leur capacité à fixer et stabiliser les HBGF (Heparin Binding Growth Factors) et à bloquer un certain nombre de protéases matricielles.

Or, il a été montré que les héparanes sulfates interviennent de façon primordiale dans les événements régulant l'adhésion des cellules circulantes à la paroi vasculaire et dans leur pénétration des sites inflammatoires [3]. En effet, des animaux déficients en une enzyme de sulfatation des héparanes sulfates, et donc possédant des héparanes sulfates non fonctionnelles, présentent une inhibition de l'expression de la L-sélectine impliquée dans les phénomènes de margination, de ralentissement et de rolling qui précédent la diapédèse des cellules circulantes. Sur ces mêmes animaux, le transfert des chimiokines exprimées dans le milieu périvasculaire vers la surface interne des vaisseaux est altéré et le guidage des cellules circulantes diminué. On sait par ailleurs que l'héparine (prototype des héparanes sulfates) a une action inhibitrice similaire à l'anomalie de ces animaux, sur la pénétration des cellules inflammatoires, probablement par un phénomène de compétition moléculaire, qui entraine des anomalies identiques à celles observées chez les animaux déficients en une enzyme de sulfatation des héparines sulfates ${ }^{[4]}$.

De plus, un certain nombre de molécules - le TNF alpha, IL 6, RANKL et OPG - peuvent se lier aux héparanes sulfates. Or, ces molécules sont impliquées dans la différenciation, l'activité et la survie des ostéoclastes. Les RGTA pourraient donc interférer avec l'activité de ces molécules et l'ensemble de ces phénomènes expliqueraint en partie les résultats observés sur le modèle d'inflammation parodontale.

Le modèle animal utilisé pour cette étude est un modèle de remaniement osseux synchronisé mandibulaire consécutif à l'extraction des molaires maxillaires antagonistes chez le rat [5]. Sur ce modèle, on sait que le pic de résorption a lieu quatre jours après les extractions. Ce modèle permet donc dans un temps très court de pouvoir étudier toutes les étapes de la réaction inflammatoire conduisant à la résorption osseuse :

1. Agression (extraction des molaires maxillaires)

2. Relargage des médiateurs dans le périoste mandibulaire

3. Arrivée des cellules circulantes dans le site 
4. Recrutement et migration des précurseurs ostéoclastiques

5. Différenciation des ostéoclastes

6. Résorption osseuse

Le but de l'étude est de déterminer à quels stades interviennent les RGTA dans cette cascade d'événements en étudiant plus particulièrement l'hypothèse selon laquelle les RGTA interagiraient avec les molécules (sélectines) induisant l'arrêt des leucocytes circulants sur l'endothélium vasculaire.

\section{MATÉRIEL ET MÉTHODE}

Trente trois rats Wistar mâles de $300 \pm 20 \mathrm{~g}$, âgés de 7 semaines (Janvier, France), ont été utilisés pour cette étude pratiquée en respectant les directives de la Commission européenne sur l'expérimentation animale. Ces animaux ont été anesthésiés par voie intrapéritonéale à l'aide de chloral à $8 \%$ hydraté (Merck, France) pour pratiquer les extractions des trois molaires maxillaires droites. Puis ces animaux sont divisés en 4 groupes:

- Groupe 1 ( $n=9$ ) : SHAM (pour imposteur) 10H Les animaux ont été traités avec une injection intra-musculaire de sérum physiologique 10 heures après les extractions.

- Groupe $2(n=8)$ : RGTA 10H

Les animaux ont été traités avec une injection intra-musculaire de RGTA (1,5 mg/kg) 10 heures après les extractions.

- Groupe $3(n=10)$ : SHAM $11 \mathrm{H}$

Les animaux ont été traités avec une injection intra-musculaire de sérum physiologique 11 heures après les extractions.

- Groupe $4(n=6)$ : RGTA 11H

Les animaux ont été traités avec une injection intra-musculaire de RGTA $(1,5 \mathrm{mg} / \mathrm{kg}) 11$ heures après les extractions.

Le choix des deux temps d'injection, 10 heures et 11 heures, s'est fait en fonction de la cinétique du modèle connue avec précision [6,7]. On sait que la dégranulation des mastocytes intervient 9 heures après les extractions. Ce phénomène va engendrer le recrutement des cellules inflammatoires et des précurseurs des ostéoclastes. Cette cinétique précise également que le recrutement est maximal 12 heures après l'agression mécanique. II semble alors judicieux de tenter de bloquer ce recrutement en injectant le RGTA entre ces deux temps clés.

La molécule utilisée dans cette étude, le RGTA OTR4120 est un carboxyméthyl sulfate dextrane obtenu à partir d'un dextrane natif T40. Pour obtenir ce RGTA, certains groupes hydroxyl des résidus dextrane glucose du dextran T40 sont substitués de façon contrôlée par des groupements carboxyméthyl, puis des groupements benzylamide et/ou O-sulfonate sont rajoutés ${ }^{[8]}$. Le RGTA purifié est ensuite ultrafiltré et sa composition chimique exacte est obtenue par microanalyse, spectrophotométrie et résonance magnétique nucléaire.

Tous les animaux ont été sacrifiés après anesthésie au chloral $8 \%$, par exsanguination cardiaque, 12 heures après l'extraction des molaires maxillaires droites. Les hémi-mandibules antagonistes ont été prélevées et immergées dans de l'alcool à $70 \%$ à $4{ }^{\circ} \mathrm{C}$, puis progressivement déshydratées par passages successifs dans des bains d'alcool de concentration croissante. Ces hémi-mandibules ont été incluses, sans déminéralisation, dans du méthacrylate de méthyle, résine polymérisant à froid $\left(-20^{\circ} \mathrm{C}\right)$. Des coupes de $4 \mu \mathrm{m}$ d'épaisseur (Microtome Polycut E Leica, Allemagne) de la zone d'intérêt ont été réalisées dans le plan horizontal, perpendiculaire à l'axe des racines des molaires.

La zone d'intérêt est identifiée par l'apparition de la crête alvéolaire vestibulaire (en regard de la joue) à hauteur de la première molaire. En effet, l'induction de la résorption qui fait suite à l'extraction des molaires antagonistes s'opère dans une zone bien précise de la surface périostée. Elle s'étend de la partie distale de la racine mésiale de la première molaire à la face distale de cette dent (Fig. 1).

Une coloration histologique de routine (bleu de toluidine à 1\%, pH 3.8) a d'abord été réalisée permettant de compter les leucocytes à proximité de la zone en résorption. L'utilisation d'un anticorps monoclonal biotinylé de souris anti-ED1 (Chemicon, France) a permis le marquage et le comptage des cellules de la lignée monocytes/macrophages, à laquelle appartiennent les médecine

buccale

chirurgie

buccale

VOL. $14, \mathrm{~N}^{\circ} 3$ 2008

page 163 


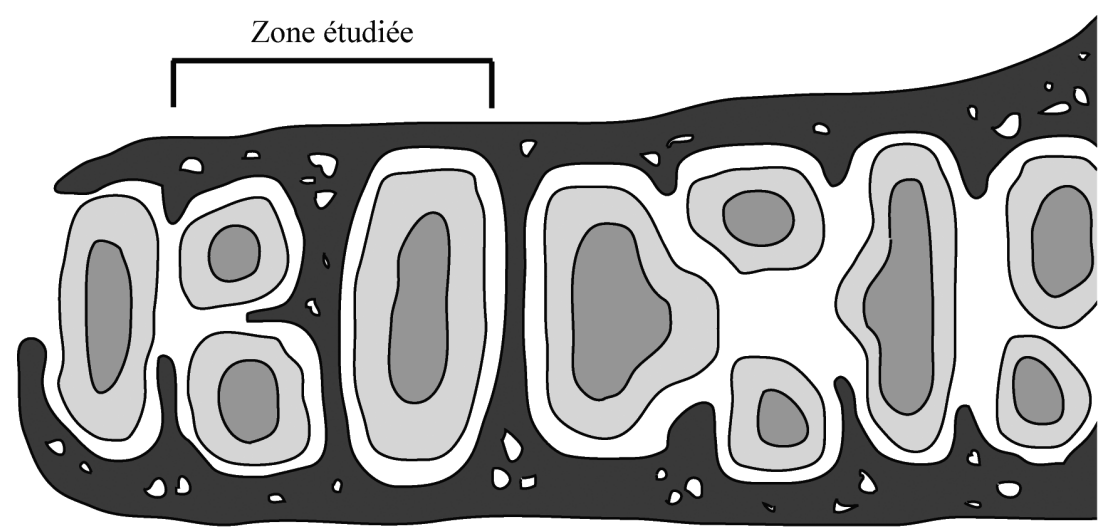

Première molaire

Deuxième molaire

Figure 1 : Vue schématique sur une coupe $(x 40)$ des limites anatomiques de la zone étudiée. Schematic view of the area of interest $(x 40)$ and anatomical limits.

médecine

buccale

chirurgie

buccale

VOL. $14, \mathrm{~N}^{\circ} 3$ 2008

page 164 précurseurs ostéoclastiques et les ostéoclastes. La révélation des sites marqués est faite à l'aide d'un complexe avidine biotine peroxydase (ABC Vectastain, Vector, USA). Les cellules contenant un marquage marron intracytoplasmique sont considérées comme positives (ED1+).

Tous les comptages cellulaires ont été réalisés à l'aide d'un analyseur d'image semi-quantitatif informatique, couplé à un microscope (Zeiss, Allemagne) et à une caméra vidéo (Sony, Japon), à grandissement constant $(x 200)$.

Les données ont été comparées à l'aide du test non paramétrique de Kruskall-Wallis pour déterminer les variations intergroupes. Quand ce test était significatif, les groupes ont été comparés deux à deux par le test $U$ de Mann-Whitney. Les

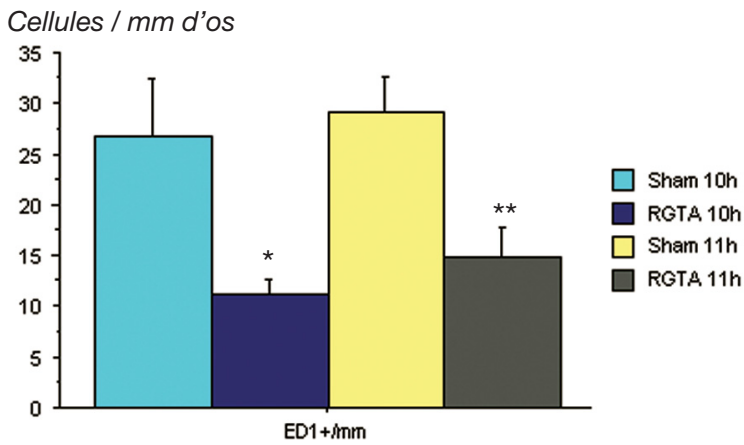

Figure 2 : Nombre de cellules ED1+ par mm d'os dans la zone de résorption dans les quatre groupes $\left(^{*}: \mathrm{p}<0.02 ;{ }^{* *}: \mathrm{p}<0.01\right)$.

Number of ED1+ cells per $\mathrm{mm}$ of bone surface in the resorption area in the four groups of animals $\left(^{*}: p<0.02\right.$; $\left.{ }^{* *}: p<0.01\right)$. différences ont été données comme significatives à moins de $5 \%(p<0,05)$. Les résultats sont donnés \pm ESM (erreur standard moyenne).

\section{RÉSULTATS}

D'une part, on constate que le nombre des monocytes/macrophages marqués ED1+, présents dans le site inflammatoire, diminue de moitié entre les animaux contrôles (SHAM) et les animaux traités au RGTA 10 heures et 11 heures après I'induction du modèle $\left({ }^{*}: p<0.02 ;{ }^{* *}: p<0.01\right)$. En revanche, le nombre des leucocytes totaux recrutés n'est pas diminué de façon significative par le traitement (Fig. 2, 3, 4A-4D).

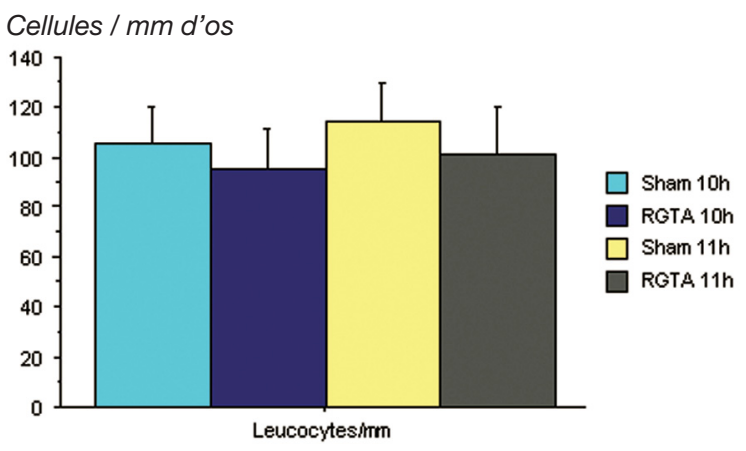

Figure 3 : Nombre de leucocytes par mm d'os dans la zone de résorption dans les quatre groupes.

Number of leucocyte cells per $\mathrm{mm}$ of bone surface in the resorption area in the four groups of animals. 


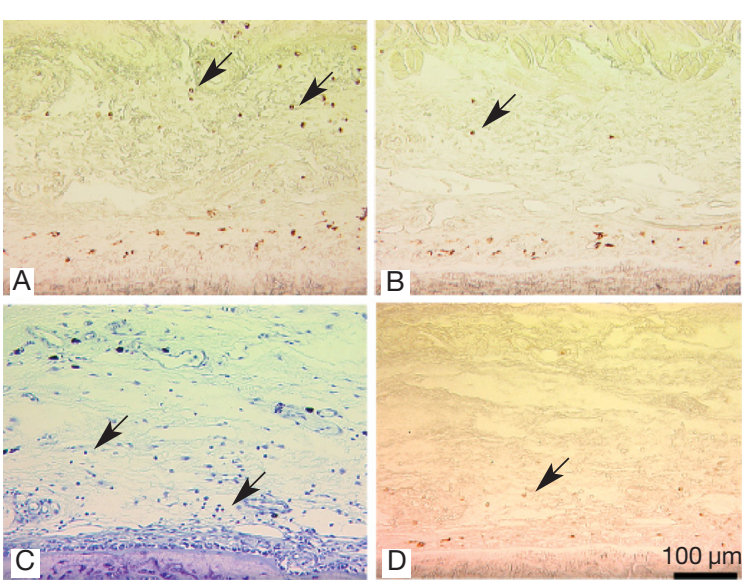

Figure 4 A : Mise en évidence des cellules ED1+ (flèches) chez un animal SHAM (grossissement x200). ED1+ cells (arrows) in a SHAM animal (magnification $x 200)$.

Figure 4B : Mise en évidences des cellules ED1+ (flèches) chez un animal traité; le nombre de cellules marquées est bien inférieur par rapport à celui de l'animal SHAM de la figure A (grossissement x200).

$E D 1+$ cells (arrows) in a treated animal; the number of positive cells is lower than in the SHAM animal showed in figure $A$ (magnification $x 200)$.

Figure 4C : Coloration au bleu de toluidine permettant de quantifier les leucocytes (flèches) (grossissement x200).

Toluidine blue staining allowing the quantification of leucocytes (arrows) (magnification x200).

Figure 4D : Immunohistochimie pour les cellules ED1+ chez le même animal que celui de la figure $\mathrm{C}$; la comparaison des deux images montrant la même zone met bien en évidence la faible proportion des cellules ED1+ parmi l'ensemble des cellules inflammatoires (grossissement $\mathrm{x} 200$ ).

Immunohistochemical staining of the ED1+ cells in the same animal than in the figure C; comparison of the two figures showing the same area illustrates the weak number of ED1+ cells in the whole inflammatory cells (magnification x200).

D'autre part, lorsqu'on calcule le rapport du nombre des cellules ED1+ sur le nombre de leucocytes totaux, on constate que la proportion des monocytes/macrophages au sein des leucocytes est également pratiquement diminuée de moitié lors de l'utilisation du RGTA. Sur ce point la différence est uniquement significative lorsque le traitement est injecté 10 heures après les extractions des dents maxillaires $\left(^{\star \star}: p<0.01\right)$ (Fig. 5).

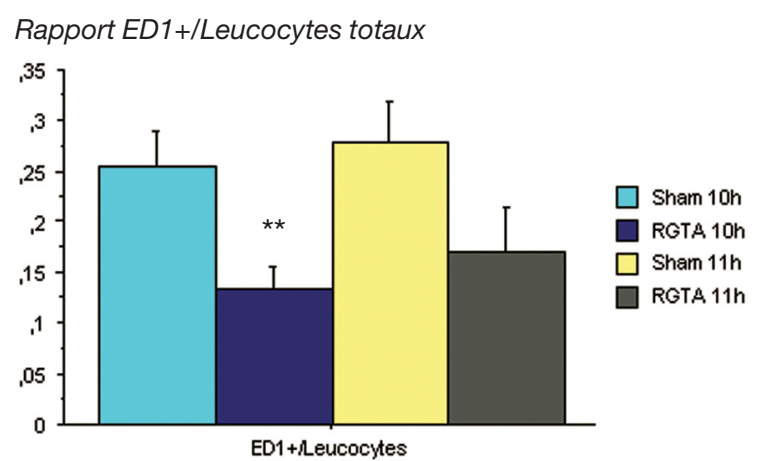

Figure 5 : Evaluation du rapport du nombre de cellules $\mathrm{ED1}$ + par mm d'os sur le nombre de leucocytes totaux par $\mathrm{mm}$ d'os dans la même zone de résorption $\left({ }^{*}: p<0.01\right)$.

Ratio of the number of ED1+ cells on the number of whole leucocytes in the same area of resorption $(* *: p<0.01)$.

\section{DISCUSSION}

Ce travail a montré que le recrutement des monocytes ED1+, considérés comme de futurs préostéoclastes, est diminué de façon significative par l'action du RGTA. La diminution est plus marquée quand l'injection a lieu 10 heures après les extractions. On peut donc penser que le recrutement de ces cellules démarre rapidement après la dégranulation des mastocytes qui a lieu 9 heures après les extractions. On peut alors penser qu'une injection plus précoce du produit entraînerait un blocage plus efficace de la sortie des cellules. En toute logique, on peut également affirmer que l'injection 11 heures après les extractions permet aux cellules d'être recrutées normalement entre 9 et 11 heures et que le RGTA ne peut agir que durant une heure, soit la moitié du temps par rapport au groupe 10 heures.

Paradoxalement, le nombre des leucocytes totaux sortant de la circulation sanguine n'est pas diminué par le traitement. La proportion du nombre de monocytes parmi l'ensemble des leucocytes est donc diminuée par le traitement.

L'action du RGTA semble entraîner un mécanisme de filtration cellulaire et de recrutement spécifique d'un type de leucocyte ou d'un autre lors de la diapédèse. Ici, le RGTA inhiberait un mécanisme de recrutement cellulaire spécifique aux monocytes. médecine

buccale chirurgie buccale

VOL. $14, \mathrm{~N}^{\circ} 3$ 2008

page 165 


\section{CONCLUSION ET PERSPECTIVES}

Les premiers résultats de cette étude tendent à montrer que le RGTA, mimétique des héparanes sulfates, perturbe le recrutement des différentes classes de leucocytes circulants. Ce travail préliminaire a ouvert plusieurs perspectives de recherche pour tenter de comprendre pourquoi le nombre de monocytes est diminué par le RGTA. II faudra étudier le recrutement des leucocytes étape par étape pour pouvoir apprécier avec précision la ou les actions du RGTA, et notamment toutes les étapes faisant intervenir les héparanes sulfates. Enfin, il faudra également confirmer que

\section{RÉFÉRENCES}

médecine buccale chirurgie buccale

VOL. $14, \mathrm{~N}^{\circ} 3$ 2008

page 166
1 - Barritault D, Caruelle JP. Les agents de régénération (ou RGTAs) : une nouvelle approche thérapeutique. Ann Pharm Fr 2006 ; 64 : 135-44.

2 - Escartin Q, Lallam-Laroye C, Baroukh B, Morvan FO, Caruelle JP, Godeau G, Barritault D, Saffar JL. A new approach to treat tissue destruction in periodontitis with chemically modified dextran polymers. FASEB J 2003 ; $17: 644-51$.

3 - Wang L, Fuster M, Sriramarao P, Esko JD. Endothelial heparan sulfate deficiency impairs L-selectin- and chemokine-mediated neutrophil trafficking during inflammatory responses. Nat Immunol $2005 ; 6$ : 861-2.

4 - Nelson RM, Cecconi O, Roberts G, Aruffo A, Linhardt RJ, Bevilacqua MP. Heparin oligosaccharides bind Land P-selectin and inhibit acute inflammation. Blood $1993 ; 82$ : 3253-8.

5 - Tran Van P, Vignery A, Baron R. An electron-microscopic study of the bone-remodeling sequence in the rat. Cell Tissue Res 1982 ; 225 : 283-92. la chute du nombre de cellules ED1+ est bien corrélée à une diminution de la résorption osseuse. Pour poursuivre l'étude du recrutement des leucocytes, monocytes, granulocytes et lymphocytes, étape par étape, et pour pouvoir apprécier avec précision la ou les actions du RGTA, le protocole décrit ci-dessus a été repris. Malheureusement, cette nouvelle étude n'a pas permis de confirmer l'ensemble des résultats précédents et, de plus, on a constaté que les résultats n'étaient absolument pas reproductibles; le modèle expérimental a donc été abandonné. Ce travail a néanmoins permis de progresser dans le domaine de connaissance des RGTA.

6 - Baroukh B, Cherruau M, Dobigny C, Guez D, Saffar JL. Osteoclasts differentiate from resident precursors in an in vivo model of synchronized resorption: a temporal and spatial study in rats. Bone $2000 ; 27: 627-34$.

7 - Fouilloux I, Biosse Duplan M, Baroukh B, Cherruau M, Saffar JL, Lesclous P. Mast cell activation and degranulation occur early during induction of periosteal bone resorption. Bone 2005 ; 38 : 59-66.

8 - Ledoux, D, Papy-Garcia D, Escartin Q, Sagot M A, Cao Y, Barritault D, Courtois J, Hornebeck W, Caruelle JP. Human plasmin enzymatic activity is inhibited by chemically modified dextrans. J Biol Chem $2000 ; 275$ : 29383-90.

Remerciements : Les auteurs tiennent à remercier Mesdames Brigitte Baroukh et Annie LLorens, techniciennes de laboratoire, pour leur assistance technique ainsi que la Société Francophone de Médecine Buccale et Chirurgie Buccale pour son soutien financier. 